\title{
RADIO FREQUENCY INTERFERENCE DETECTION FROM SPACE
}

\author{
WILLIAM E. HOWARD III \\ U.S. Naval Space Command Dahlgren, Virginia 22448-5107
}

\section{THE PROBLEM AND ITS SOLUTION}

Occasional interference experienced in the channels of communications satellites has prompted an analysis to see how radio frequency interference (RFI) might be detected from space. RFI may be experienced on any type of satellite, including commercial and scientific satellites. For a satellite in geostationary orbit that interference may come from anywhere in the hemisphere under the satellite. Because the location of an interfering transmitter is so uncertain, traditional means for geolocating it is not effective. "Down-looking" detectors are needed to detect the "up-looking" interference. Moreover, a low cost, simple solution to the problem - one in which the cost to geolocate is small relative to the cost of the downtime in the channel - is required in order to make the solution tractable.

A demonstration is being planned that will detect RFI by means of a radio interference-detecting satellite located in low earth orbit. A contract for a simple satellite called PRoFILE (Passive Radio Frequency Interference Location Experiment) has been awarded to the ARDAK Corporation, with major participation by the Fairchild Space Company. This demonstration to geolocate RFI from space will last from one to three years.

While there is a potential for interference at any satellite operating frequency, the easiest problems to address are those near $300 \mathrm{MHz}$ in the UHF band because at those relatively low frequencies the RFI-detecting satellite, the antennas for transmission between the satellite and its associated ground station and the transmitters and receivers on the satellite and at the ground station are simple and relatively inexpensive.

The satellite to be used for this mission of RFI detection is one of a class of satellites increasingly referred to as SPINSATs (Special Purpose, Inexpensive Satellites). It will use inexpensive components to keep the total cost down and it will be launched from a relatively low cost launcher into a circular, low earth orbit. It will have a receiver capable of being tuned anywhere within the UHF communications satellite band.

The ground station will be a simple personal computer, not significantly more complicated than the IBM AT class of computers, and it will have a rooftop antenna. It will be capable of automated operation, with only intermittent monitoring required by its operating personnel. 


\section{CONCEPT OF OPERATIONS}

Once PRoFILE has been launched and is in operation, if interference is experienced at a communications receiver, it is reported by the receiving personnel. The parameters characterizing the interference are relayed to the PRoFILE ground station and the satellite is tasked to geolocate the interference. PRoFILE is commanded to turn on and off at specified times that coincide with the times at which the satellite is within the line of sight to the communications satellite that is experiencing the RFI. During this time the signal is collected from the interfering transmitter and its signal characteristics are stored in the satellite memory. The signal information is downlinked to the ground station where it is processed within seconds to minutes to yield the earth coordinates of the interfering transmitter. Those coordinates are given to the proper authorities who will take the steps necessary to turn off the offender. Because of the orbital geometry of the satellite, this method is expected to locate an offending transmitter within one to twelve hours of initial tasking.

The geolocation method essentially uses a Doppler technique that is the inverse of the one employed by commercial users of the U.S. Navy's TRANSIT satellite system (Ref 1 ). The accuracy expected is of the order of two to twenty kilometers for CW signals.

\section{COST AND TIME SCALE}

The cost of PRoFILE will be in the range $\$ 1-3$ million, including the satellite and the ground station, exclusive of the cost to launch it. If it is launched by a "Get-Away Special (GAS can)" on the space shuttle, the launch cost will be under $\$ 50,000$. Estimated annual costs for routine operation of the ground station are in the neighborhood of $\$ 200,000$ or less. The time required to build the satellite is less than two years and it is expected to be ready to launch before 1992 . We note that at that time the sunspot cycle will be near a maximum, increasing the atmospheric drag on the satellite. To achieve a lifetime in low earth orbit that exceeds one year, PRoFILE will need to achieve an altitude of at least 250 nautical miles.

\section{THE FUTURE ?}

The research and development effort described here promises to lead to more and more routine detection of radio frequency interference from space using simple, SPINSAT-type spacecraft. Beginning at the UHF band, the techniques can be developed for later use in the SHF and EHF bands. Once the longer-term interference problems have been addressed, we may turn our attention to the problems and causes of shorter-term interference and develop more useful data bases that will lead to better quantification of the types and sources of interference of all types and on all time scales. 


\section{REFERENCES}

1. Stansell, Thomas A., The Transit Navigation System (Status, Theory, Performance and Applications), Magnavox Advanced Products and Systems Company R-5933A, June 1983.

See also "Artificial Earth Satellites", The Johns Hopkins University/Applied Physics Laboratory, SDO 1600 (Revised) May 1987. 